\title{
Langmuir-Maxwell and Langmuir-Smoluchowski boundary conditions for thermal gas flow simulations in hypersonic aerodynamics
}

\author{
Nam T.P. Le ${ }^{a}$, Craig White ${ }^{b}$, Jason M. Reese ${ }^{b, *}$, Rho S. Myong ${ }^{a, *}$ \\ ${ }^{a}$ Research Center for Aircraft Parts Technology, and Department of Aerospace and System Engineering, Gyeongsang National University, Jinju 660-701, South Korea \\ ${ }^{\mathrm{b}}$ Department of Mechanical and Aerospace Engineering, University of Strathclyde, Glasgow G1 1XJ, United Kingdom
}

\section{A R T I C L E I N F O}

\section{Article history:}

Received 1 September 2011

Received in revised form 9 April 2012

Accepted 21 April 2012

Available online 11 June 2012

\section{Keywords:}

Langmuir-Maxwell

Langmuir-Smoluchowski

New boundary conditions

Adsorption

Hypersonics

Simulation results

\begin{abstract}
A B S T R A C T
The simulation of nonequilibrium thermal gas flow is important for the aerothermodynamic design of re-entry and other high-altitude vehicles. In computational fluid dynamics, the accuracy of the solution to the Navier-Stokes-Fourier (N-S-F) equations depends on the accuracy of the surface boundary conditions. We propose new boundary conditions (called the Langmuir-Maxwell and the Langmuir-Smoluchowski conditions), for use with the N-S-F equations, which combine the Langmuir surface adsorption isotherm with the Maxwell/Smoluchowski slip/jump conditions in order to capture some of the physical processes involved in gas flow over a surface. These new conditions are validated for flat plate flow, circular cylinder in cross-flow, and the flow over a sharp wedge for Mach numbers ranging from 6 to 24, and for argon and nitrogen as the working gases. Our simulation results show that the new boundary conditions give better predictions for the surface pressures, compared with published experimental and DSMC data.
\end{abstract}

(c) 2012 Elsevier Ltd. All rights reserved.

\section{Introduction}

Translational nonequilibrium of a rarefied gas flow can be characterized by the Knudsen number, $K n$, which is the ratio of the gas molecular mean free path $\lambda$ (i.e. the average distance a molecule travels between successive intermolecular collisions) to a characteristic length of an aerodynamic vehicle body, $l$. In order for the Navier-Stokes-Fourier (N-S-F) equations, as generally used in Computational Fluid Dynamics (CFD) to produce reasonable results in the $K n$ range up to 0.1 , velocity slip and temperature jump boundary conditions should be applied at the surface.

The first-order conventional slip condition was developed by Maxwell for a flat plate [1]. It remains the simplest and most useful description of the slip condition, and depends on the normal gradient of velocity at the surface and a thermal creep term. The firstorder slip condition described by Shen et al. [2] was derived using the Chapman-Enskog solution of the Boltzmann equation under the relaxation time approximation. In this condition, the slip velocity depends not only on the velocity gradient in the surface-normal direction but also on the pressure gradient in the flow direction. A general slip condition for a solid surface in rarefied multi-compo-

\footnotetext{
* Corresponding authors. Tel.: +44 1415483131 (J.M. Reese), tel.: +82 55772 1645 (R.S. Myong).

E-mail addresses: namle@gnu.ac.kr (N.T.P. Le), craig.white@strath.ac.uk (C. White), jason.reese@strath.ac.uk (J.M. Reese), myong@gnu.ac.kr (R.S. Myong).
}

nent gas flows has been reported by Zade et al. [3]. The slip condition developed by Gökçen and MacCormack [4] aims to reduce to the classical Maxwell slip condition in the continuum limit, and yield the correct shear stress in free molecular flow in the limiting case of very large Knudsen numbers. All these first-order slip conditions make use of a free parameter in the slip equation, namely the tangential momentum accommodation coefficient $\sigma_{u}$. This varies between 0 and 1 , and determines the proportion of molecules reflected from the surface specularly $\left(1-\sigma_{u}\right)$ or diffusely $\sigma_{u}$.

Second-order slip boundary conditions have also been developed [5-8]. These include an additional second-order term in the normal gradient of velocity. Our simulation results in [9] showed that using the second-order slip condition is not much better than using the conventional Maxwell slip condition, when simulating thermal gas flows in hypersonic aerodynamics.

The temperature jump condition developed by Smoluchowski [10] is driven by the heat flux to the surface in the normal direction. A general temperature jump condition for rarefied multicomponent gas flows is also described by Zade et al. [3]. Gökçen and MacCormack [4] proposed a temperature jump condition both to simplify the Smoluchowski jump condition, and to yield the correct heat transfer in free molecular flow in the limiting case of very large Knudsen numbers. These temperature jump conditions also depend on a free parameter, namely the thermal accommodation coefficient $\sigma_{T}$, which varies between 0 and 1 , with 1 for a perfect energy exchange between gas molecules and solid surfaces, and 0 corresponding to no energy exchange. 


\begin{tabular}{|c|c|c|c|}
\hline \multicolumn{4}{|c|}{ Nomenclature } \\
\hline $\bar{v}$ & mean molecular velocity & $T_{S}$ & constant temperature \\
\hline I & identify tensor & $x$ & the running distance from the tip of the flat plate \\
\hline $\mathbf{n}$ & surface normal vector & $\mathbf{u}$ & velocity \\
\hline $\mathbf{Q}$ & heat flux along the surface & & \\
\hline $\mathbf{q}$ & heat flux & \multicolumn{2}{|c|}{ Greek symbols } \\
\hline S & transformation tensor & $\alpha$ & fraction of gas molecular coverage on a surface \\
\hline $\operatorname{tr}$ & trace & $\beta$ & an equilibrium constant \\
\hline$a$ & a coefficient specific to each boundary conditions & $\Pi$ & stress tensor at the surface \\
\hline$A_{m}$ & mean area of a site & $\eta$ & number density of molecules \\
\hline$A_{S}$ & constant for Sutherland's law & $\gamma$ & specific heat ratio \\
\hline$C_{\Delta}$ & coefficient & $\Gamma_{n}$ & total number of molecules issuing effusively through a \\
\hline$c_{p}$ & specific heat of a gas at constant pressure & & hole \\
\hline$c_{v}$ & specific heat of a gas at constant volume & $\lambda$ & molecular mean free path \\
\hline d & molecular diameter & $\mu$ & dynamic viscosity \\
\hline$d_{B}$ & distance from the cell centre to the boundary face centre & $\nabla$ & gradient \\
\hline$D_{e}$ & heat of adsorption & $\nabla_{\mathbf{n}}$ & gradient normal to the boundary surface \\
\hline$e^{e}$ & internal energy & $\Phi$ & cylinder angle \\
\hline$H$ & height of the base & $\phi$ & variable of interest, e.g. velocity or temperature \\
\hline$k$ & thermal conductivity & $\Phi_{L}$ & limiting no-slip/jump value of $\phi$ \\
\hline$K_{L}$ & a chemical equilibrium constant & $\rho$ & density \\
\hline$K n$ & Knudsen number & $\sigma$ & fraction coefficient \\
\hline$L$ & length of the top wedge surface & $\sigma_{T}$ & thermal accommodation coefficient \\
\hline$M$ & chemical name of gas molecules & $\sigma_{u}$ & tangential momentum accommodation coefficient \\
\hline$N_{A}$ & Avogadro number & $\tau$ & shear stress \\
\hline$p$ & gas pressure at the surface & $\Pi_{\mathrm{mc}}$ & curvature effect \\
\hline $\operatorname{Pr}$ & Prandtl number & & \\
\hline$R$ & specific constant of gas & \multicolumn{2}{|c|}{ Super and subscripts } \\
\hline$R_{u}$ & universal gas constant & $\infty$ & freestream condition \\
\hline$S$ & distance along the wedge surface & $\mathrm{T}$ & transpose \\
\hline$S_{v}$ & a vacant surface & $w$ & surface \\
\hline$S M$ & an occupied surface & & \\
\hline$T$ & temperature & & \\
\hline
\end{tabular}

Alternative slip velocity and temperature jump conditions that overcome the problem of free parameters were proposed by Myong and co-workers [11,12]. These are the so-called 'Langmuir conditions', based on the Langmuir adsorption isotherm. They give good results for some rarefied gas microflows. However, the problem when applying these conditions is the choice of reference values for either the velocity or the temperature, which depend on the particular case being considered. The reference values might be evaluated either a mean free path away from the surface, or in the freestream. Our simulation results in [9] show that the Langmuir conditions did not give particularly good results for thermal gas flows in hypersonic aerodynamics.

Comparing the Langmuir conditions with the Maxwell/Smoluchowski boundary conditions, it is noted that (a) the adsorption of gas on a solid surface is ignored in developing the Maxwell/ Smoluchowski boundary conditions, which consider only kinds of diffuse reflection $[13,14]$, and (b) the Langmuir boundary conditions only consider the adsorption of gas on to a solid surface, and lack the kinetic theory component of the Maxwell/Smoluchowski conditions.

In this paper new boundary conditions are proposed that combine the Langmuir adsorption isotherm with the Maxwell/Smoluchowski conditions, with the aim of achieving a more realistic physical fluid model. The new boundary conditions address the lack of the physical adsorption of gas within the Maxwell/Smoluchowski boundary conditions, and the problem of the reference values required by the Langmuir boundary conditions. The present work focuses on surface boundary conditions for CFD using the $\mathrm{N}-\mathrm{S}-\mathrm{F}$ equations, and for low-enthalpy flows where chemical nonequilibrium is not important.

\section{Nonequilibrium boundary conditions}

Most nonequilibrium slip/jump boundary conditions can be expressed in the general form:

$\phi+a \nabla_{\mathbf{n}}(\mathbf{S} \cdot \phi)=\Phi_{L}$,

where bold type denotes a vector or tensor quantity; $\nabla_{\mathbf{n}} \equiv \mathbf{n} \cdot \nabla$ is the component of the gradient normal to the boundary surface, with $\mathbf{n}$ being the unit normal vector defined as positive in the direction pointing out of the flow domain; $\Phi_{L}$ is the limiting value in the case of no-slip/jump, i.e. the surface velocity or temperature. The tensor $\mathbf{S}=\mathbf{I}-\mathbf{n n}$ removes normal components of any non-scalar field, e.g. velocity, so that slip only occurs in the direction tangential to the surface. The normal gradient can be expressed numerically as:

$\nabla_{\mathbf{n}} \phi=C_{\Delta}\left(\phi-\phi_{i}\right)$

where the subscript ' $i$ ' denotes a value in the numerical cell adjacent to the boundary face of the solid surface, and $c_{\Delta}=1 /\left|d_{B}\right|$, with $d_{B}$ the distance from the numerical cell centre to the boundary face centre of the solid surface.

In this paper, all nonequilibrium boundary conditions will be expressed in the form of the general Eq. (1) above. The common Maxwell slip velocity and Smoluchowski temperature jump conditions are now revisited.

\subsection{Slip velocity}

In gas-surface interactions, the gas molecules can be grouped into two streams relative to the surface: approaching and receding. 
The viscous drag on the surface is due to the difference between the tangential momentum of the approaching stream and that carried away by the receding stream. The tangential momentum of the gas molecules is responsible for the shear stress, $\tau$. Maxwell makes the assumption that the approaching gas molecules and the receding gas molecules have equal influence on the total shear stress at the surface [14]:

$\left[\frac{1}{2} \tau\right]_{\text {approaching }}+\left[\frac{1}{2} \tau\right]_{\text {receding }}=[\tau]_{\text {total }}$.

The stream of molecules receding from the surface is assumed to be equivalent to a simple effusive flow. A typical effusive velocity distribution will be that of a flow of individual molecules through a small hole in a planar surface with diameter of the order of the mean free path. The total number of molecules that would issue effusively, $\Gamma_{n}$, may be expressed in terms of the mean molecular velocity, $\bar{v}$, and the number density of molecules in the flow, $\eta$, as [14]:

$\Gamma_{n}=\frac{1}{4} \eta \bar{v}$

This number of molecules leaving the surface is then multiplied by the slip velocity at the surface, $\mathbf{u}$, to produce the receding stream's contribution to the total shear stress at the surface. The observation of a slip velocity means that some tangential momentum is retained by the gas at the surface. To determine the slip velocity while conserving momentum, Maxwell introduced the tangential momentum accommodation coefficient, $\sigma_{u}$. Incorporating density $\rho=\eta m$, Eq. (3) becomes:

$\sigma_{u}\left[\frac{1}{2} \tau+\frac{1}{4} \rho \bar{v} \mathbf{u}\right]=\tau$,

which can be re-arrange as,

$\mathbf{u}=2\left(\frac{2-\sigma_{u}}{\sigma_{u}}\right) \frac{\tau}{\rho \bar{v}}$

The mean molecular velocity, $\bar{v}$, from a Maxwellian equilibrium distribution [14] is

$\bar{v}=2 \sqrt{(2 R T / \pi)}$.

There are a number of different definitions of the mean free path $\lambda$; here, the Maxwellian definition is used, i.e.

$\lambda=\frac{\mu}{\rho} \sqrt{\frac{\pi}{2 R T}}$.

From Eqs. (6)-(8), the most common form of the Maxwell slip boundary condition in rarefied gas flows is then obtained [1]:

$\mathbf{u}=\left(\frac{2-\sigma_{u}}{\sigma_{u}}\right) \frac{\lambda \tau}{\mu}$

This simple formulation neglects the thermal creep, a process whereby a temperature gradient tangential to the surface generates additional slip flow in the direction of increasing temperature. The gas would then slide over a surface from colder to hotter regions. Maxwell's general equation for use in three-dimensional geometries, including thermal creep, can be written in tensor form by replacing $\tau=-\mathbf{S} \cdot(\mathbf{n} \cdot \boldsymbol{\Pi})$ as follows:

$\mathbf{u}=-\left(\frac{2-\sigma_{u}}{\sigma_{u}}\right) \frac{\lambda}{\mu} \mathbf{S} \cdot(\mathbf{n} \cdot \boldsymbol{\Pi})-\frac{3}{4} \frac{\operatorname{Pr}(\gamma-1)}{\gamma p} \mathbf{q}+\mathbf{u}_{w}$,

where the heat flux is $\mathbf{q}=\mathbf{Q} \cdot \mathbf{S}$ at the surface.

Eq. (10) can be expressed in the general form of Eq. (1) by substituting $\boldsymbol{\Pi}=\mu \nabla \mathbf{u}+\Pi_{\mathrm{mc}}$, with $\boldsymbol{\Pi}_{\mathrm{mc}}=\mu(\nabla \mathbf{u})^{T}-\left(\frac{2}{3}\right) \operatorname{Itr}(\nabla \mathbf{u})$. Noting that $\mathbf{S} \cdot \nabla_{\mathbf{n}} \phi \equiv \nabla_{\mathbf{n}}(\mathbf{S} \cdot \phi)$

Eq. (10) then becomes: $\mathbf{u}+\left(\frac{2-\sigma_{u}}{\sigma_{u}}\right) \lambda \nabla_{\mathbf{n}}(\mathbf{S} \cdot \mathbf{u})=\mathbf{u}_{w}-\left(\frac{2-\sigma_{u}}{\sigma_{u}}\right) \frac{\lambda}{\mu} \mathbf{S} \cdot\left(\mathbf{n} \cdot \Pi_{\mathrm{mc}}\right)-\frac{3}{4} \frac{\mu}{\rho} \frac{\mathbf{S} \cdot \nabla T}{T}$

The right hand side of Eq. (11) contains three terms that are associated with (in order) the surface velocity, the so-called curvature effect [5], and thermal creep.

\subsection{Temperature jump}

Experimental observations show that the temperature of a rarefied gas at a surface is not equal to the surface temperature, $T_{w}$. The difference is called the 'temperature jump' and is driven by the heat flux normal to the surface. By analogy with Maxwell's arguments for the slip velocity, the energy of approaching molecules impinging on a unit area of surface is responsible for the heat conducted through the surface. Smoluchowski assumes that the approaching stream of molecules conducts the same level of heat (and does dissipative work) as that within the region of gas some distance away from the boundary. The approaching and receding streams contribute to half of the conduction of heat. Similar to the derivation of slip velocity, to determine the temperature jump while conserving energy a thermal accommodation coefficient, $\sigma_{T}$, is introduced (with $0 \leqslant \sigma_{T} \leqslant 1$ ). Perfect energy exchange between the gas and the solid surface corresponds to $\sigma_{T}=1$, and no energy exchange to $\sigma_{T}=0$. Therefore we have [14]:

$-\left[k \nabla_{\mathbf{n}} T\right]_{\text {total }}=\sigma_{T}\left(-\left[\frac{1}{2} k \nabla_{\mathbf{n}} T\right]_{\text {approaching }}+\left[\frac{1}{2} k \nabla_{\mathbf{n}} T\right]_{\text {receding }}\right)$,

where thermal conductivity $k$ is calculated as,

$k=\frac{c_{p} \mu}{\operatorname{Pr}}$,

where $c_{p}=\gamma c_{v}$. The internal energy of the molecules will normally be $e=c_{v} T$ per unit mass. The translatory energy of molecules colliding with unit area of the boundary is $4 / 3$ times greater than the mean translatory energy of molecules in equilibrium at the same temperature [14]. The energy of colliding molecules is then higher by a factor $(\gamma+1) / 2$, corresponding to an energy $\left(c_{v}+R / 2\right) T$. The mass of molecules colliding with unit area of boundary is $\rho \bar{v} / 4$, so the integral energy per unit area is $\rho \bar{v}(\gamma+1) e / 8$. The conduction of heat by the receding stream is the difference between the internal energy of the gas $e$ and that of the surface $e_{w}$ and is calculated as [14]:

$\left[\frac{1}{2} k \nabla_{\mathbf{n}} T\right]_{\text {receding }}=\frac{1}{8} \rho \bar{v}(\gamma+1)\left(e-e_{w}\right)$,

which is re-arranged by replacing $\rho \bar{v}=2 \mu / \lambda, e=c_{v} T$ and $e_{w}=c_{v} T_{w}$ as,

$\left[\frac{1}{2} k \nabla_{\mathbf{n}} T\right]_{\text {receding }}=\frac{1}{4} \frac{\mu}{\lambda}(\gamma+1) c_{v}\left(T-T_{w}\right)$.

Inserting Eq. (15) into Eq. (12) gives the Smoluchowski temperature jump in rarefied gas flows, which in the form of Eq. (1) is [10,14],

$T+\frac{2-\sigma_{T}}{\sigma_{T}} \frac{2 \gamma}{(\gamma+1) \operatorname{Pr}} \lambda \nabla_{\mathbf{n}} T=T_{w}$.

\subsection{Langmuir's adsorption isotherm}

Adsorption is the process by which free gas molecules attach to a surface. The gas molecule that adsorbs is the 'adsorbate' and the surface is the 'adsorbent'. Adsorption is usually described through isotherms, that is, the amount of adsorbate on the adsorbent as a function of its pressure at constant temperature. 


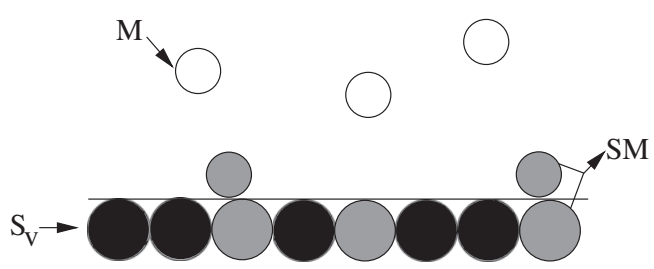

Fig. 1. Schematic of the Langmuir adsorption isotherm model.

The Langmuir adsorption isotherm model proposed for gases adsorbed on a surface, described in [11,12,17-20] is based on the following assumptions:

- adsorption cannot proceed beyond monolayer coverage of the surface;

- all surface sites are equivalent and can accommodate one adsorbed atom;

- there are no interactions between the occupied sites.

The adsorption isotherm process between the gas molecules and the surface is shown in Fig. 1. Here $M$ is the chemical name of the free gas molecules, $S_{v}$ is a vacant surface site (adsorbent) and $S M$ is an occupied surface site (adsorbate). The gas-surface interaction process can be regarded as the chemical reaction [17]:

$S_{v}+M \Longleftrightarrow S M$

If $\alpha$ is the fraction of coverage (occupied surface sites, with $0 \leqslant \alpha \leqslant 1$ ), SM is proportional to $\alpha, S_{v}$ is proportional to the number of vacant sites $(1-\alpha)$, and $M$ is proportional to the pressure of the gas, $p$. The fraction $\alpha$ has to involve the rarefaction parameter (i.e. pressure or $K n$ ), and can be calculated for different gases $[12,20]$. For a monatomic gas:

$\alpha=\frac{\beta p}{1+\beta p}$,

where $\beta$ is defined below. For a diatomic gas, it is assumed that the atoms are individually held to the metal, each atom occupying one elementary space. The rate of evaporation of the atoms is negligibly small but occasionally adjacent atoms combine together and thus nearly saturate each other chemically, so that their rate of evaporation becomes much greater. The atoms therefore leave the surface only in pairs, as molecules. Starting with a bare surface, if a small amount of gas is adsorbed the adjacent atoms will nearly always be the atoms which condensed together when a molecule was adsorbed. But from time to time two molecules will happen to be adsorbed in adjacent spaces. One atom of one molecule and one of the other may then evaporate from the surface as a new molecule, leaving two isolated atoms which cannot combine together as a molecule and are therefore compelled to remain on the surface [20]. The molecule approaching the surface may be adsorbed on the surface only if two elementary sites are vacant. The chance of one of these sites being vacant is $(1-\alpha)$ and the chance that both sites will be vacant is $(1-\alpha)^{2}$. Thus the rate of condensation is proportional to $(1-\alpha)^{2}$. Evaporation only occurs when adsorbed atoms are in adjacent sites. The chance that an atom shall be in a given site is $\alpha$ and the chance that atoms will be in adjacent sites is $\alpha^{2}$. So the rate of evaporation of molecules from the surface is proportional to $\alpha^{2}$. From this reasoning, the following adsorption isotherm for a diatomic gas can be postulated $[12,20]$ :

$\alpha=\frac{\sqrt{\beta p}}{1+\sqrt{\beta p}}$

where $\beta$ is an equilibrium constant relating to the surface temperature, given by:
$\beta=\frac{K_{L}}{R_{u} T_{w}}$

and $K_{L}$ is a chemical equilibrium constant for the reaction in Eq. (17) $[12,17]$ :

$K_{L}=A_{m} \lambda \exp \left(\frac{D_{e}}{R_{u} T_{w}}\right)$,

so that

$\beta=\frac{A_{m} \lambda}{R_{u} T_{w}} \exp \left(\frac{D_{e}}{R_{u} T_{w}}\right)$,

where $A_{m}$ is either measured or calculated approximately by $N_{A} \pi d^{2}$ / $4\left(\mathrm{~m}^{2} / \mathrm{mol}\right)$ for gases $[12,20], N_{A}=6.0221415 \times 10^{23} ; D_{e}$ is the measured value of the heat of adsorption $(\mathrm{J} / \mathrm{mol})$ : for argon and nitrogen gases $D_{e}=5255(\mathrm{~J} / \mathrm{mol})$ given in $[11,17,20]$. Pressure dependence in the fraction $\alpha$ enters through the parameter the mean-free-path $\lambda$ that includes the density parameter in Eq. (8). In order that the pressure is not cancelled out by the mean-free-path term in calculations at any iteration, the density values at the current iteration and the pressure values from the previous iteration are used for calculating the values $\lambda, \beta$ and $\alpha$. Our earlier testing of the Langmuir models with various molecular diameters [9] showed that the Langmuir models using the covalent diameter give better predictions for surface pressure and slip velocity. Therefore, the covalent diameter is used for our thermal gas flow simulations below, and is a measure of the size of the molecule which forms part of a covalent bond. The covalent diameter of argon gas is $0.21 \mathrm{~nm}$, and for nitrogen gas $0.142 \mathrm{~nm}[21]$.

\subsection{New surface boundary conditions in CFD}

In calculating the slip/jump, the Langmuir conditions only consider the molecules adsorbed on the surface. The adsorption of molecules results in a kind of diffuse reflection [13,14], and is ignored in the Maxwell/Smoluchowski slip/jump conditions, which depend on the free parameters $\sigma_{u}$ and $\sigma_{T}$. Our earlier simulation results [9] proposed the value $\sigma_{T}=1.0$ (i.e. perfect energy exchange) in the Smoluchowski jump condition. In order to conserve momentum at the surface in the Maxwell slip condition only $\sigma_{u}$ is introduced in Eq. (5). This means the molecules specularly reflected, a proportion $\left(1-\sigma_{u}\right)$, are not taken into account in the Maxwell slip condition because they conserve their incoming velocity. We propose an alternative approach for conserving momentum in the modified slip condition, that is, the molecules adsorbed should be involved in the model. Similarly, for the temperature jump condition, the molecules adsorbed should be involved in conservation of energy at the surface.

This observation suggests that only the molecules diffusively reflected and perfect energy exchange should be taken into account for developing modified slip/jump conditions. In the Maxwell/Smoluchowski conditions, this is expressed by $\sigma_{u}=\sigma_{T}=1.0$. For this reason we will compare our new conditions with other simulations that use the values $\sigma_{u}=\sigma_{T}=1.0$.

The molecules adsorbed, determined by the fraction $\alpha$, do not contribute to the component of the total fluid shear stress at the surface that is due to the receding molecules [22]. The temperature of these emitted molecules is equal to the surface temperature ( $T=T_{w}$ ), and they do not contribute to the component of the total conduction of heat at the surface that is due to the receding molecules. So following Eqs. (3) and (12), with the fraction $(1-\alpha)$ incorporated in the terms involving receding molecules, we propose:

$\left[\frac{1}{2} \tau\right]_{\text {approaching }}+\left[\frac{1}{2}(1-\alpha) \tau\right]_{\text {receding }}=[\tau]_{\text {total }}$, 
Table 1

Coefficient $a$ in Eq. (1) for various slip/jump boundary conditions.

\begin{tabular}{lll}
\hline Velocity slip & $\begin{array}{l}\text { Temperature } \\
\text { jump }\end{array}$ & Coefficient $a$ \\
\hline Maxwell & - & $\lambda\left(2-\sigma_{u}\right) / \sigma_{u}$ \\
Langmuir- & - & $\lambda /(1-\alpha)$ \\
$\quad$ Maxwell & Smoluchowski & $\lambda\left(\left(2-\sigma_{T}\right) / \sigma_{T}\right)(2 \gamma /((\gamma+1) \operatorname{Pr}))$ \\
- & Langmuir- & $(\lambda /(1-\alpha))(2 \gamma /((\gamma+1) \operatorname{Pr}))$ \\
- & Smoluchowski & \\
\hline
\end{tabular}

and

$-\left[k \nabla_{\mathbf{n}} T\right]_{\text {total }}=\sigma_{T}\left(-\left[\frac{1}{2} k \nabla_{\mathbf{n}} T\right]_{\text {approaching }}+\left[\frac{1}{2}(1-\alpha) k \nabla_{\mathbf{n}} T\right]_{\text {receding }}\right)$.

Following algebraic manipulations similar to those employed above on the Maxwell/Smoluchowski conditions, our new slip boundary condition in the form of Eq. (1) is obtained:

$\mathbf{u}+\left(\frac{1}{1-\alpha}\right) \lambda \nabla_{\mathbf{n}}(\mathbf{S} \cdot \mathbf{u})=\mathbf{u}_{w}-\left(\frac{1}{1-\alpha}\right) \frac{\lambda}{\mu} \mathbf{S} \cdot\left(\mathbf{n} \cdot \Pi_{\mathrm{mc}}\right)-\frac{3}{4} \frac{\mu}{\rho} \frac{\mathbf{S} \cdot \nabla T}{T}$,

which we term the 'Langmuir-Maxwell slip condition'. The right hand side of this new slip condition contains three terms that are associated with (in order): the surface velocity, the so-called curvature effect [5], and thermal creep. A new temperature jump condition may also be expressed in the form of Eq. (1), and termed the 'Langmuir-Smoluchowski jump condition':

$T+\frac{1}{1-\alpha} \frac{2 \gamma}{(\gamma+1) \operatorname{Pr}} \lambda \nabla_{\mathbf{n}} T=T_{w}$.

Here we have only considered physical adsorption, which implies that gases have no chemical interaction with a surface. The gases are adsorbed by the solid surface as a result of purely physical forces [23]. Therefore, the value of the heat of adsorption, $D_{e}$, is small and falls within the range from 0.1 to $10 \mathrm{kcal} / \mathrm{mol}[12,20]$. The fraction, $\alpha$, is for a surface covered by adsorbed molecules at thermal equilibrium [12]. This means that $\alpha$ has a dependency on the degree of thermal equilibrium; for example, $\alpha$ is 1 when all surfaces are covered at thermal equilibrium (i.e. when $K n \rightarrow 0$ ).

From Eqs. 11, 16, 25, and 26, the specific coefficients $a$ for the different models for velocity slip and temperature jump in the form of general Eq. (1) are collated in Table 1.

\section{Numerical case setup}

The open source CFD software OpenFOAM [24] is used in the present work. OpenFOAM uses finite volume numerics to solve systems of partial differential equations ascribed on any 3-dimensional unstructured mesh of polygonal cells. Here, the N-S-F equations are implemented and solved numerically in OpenFOAM using a finite volume discretisation and high-resolution central schemes that are described in detail in [25]. These are implemented as the solver rhoCentralFoam in OpenFOAM for simulating high speed viscous flows.

Various fundamental types of boundary conditions are implemented in OpenFOAM, including 'partial slip'; that is a mixture of a fixed value, or Dirichlet, condition and a zero gradient condition (i.e. a Neumann condition where the normal gradient is zero). The 'mixing' of the two is controlled by a fraction coefficient $\sigma$ $(0 \leqslant \sigma \leqslant 1)$ where $\sigma=0$ for the zero gradient condition and $\sigma=1$ for the fixed value condition. A reference value is also required that is assigned to the fraction of the boundary condition that is a fixed value.

For the case of scalar fields, this OpenFOAM boundary condition can be used as the basis for any slip/jump boundary condition described by Eq. (1) by setting [9]

$\sigma=1-\frac{a C_{\Delta}}{1+a C_{\Delta}}$

and setting the value of $\Phi_{L}$ in Eq. (1) to be the reference value for the fixed value component. For the case of vector fields, we modified the standard partial slip condition to include the tensor $\mathbf{S}$, so that only the tangential components of the field are 'slipped'. In either case, the fraction values $\sigma$ for the boundary conditions considered are simply determined by Eq. (27) with the values of $a$ from Table 1 .

A calorically perfect gas is considered for all simulations in the present study, so $p=\rho R T$. The Sutherland law is used for modeling the dynamic viscosity in all our CFD simulations, i.e.

$\mu=A_{S} \frac{T^{1.5}}{T+T_{S}}$

The coefficient of thermal conductivity, $k$, is then computed from Eq. (13). The values $A_{S}, T_{S}, R, \gamma$ and $\operatorname{Pr}$ of the working gases for all our CFD simulation cases are given in Table 2 [26-28].

For all simulation cases, nonequilibrium boundary conditions are applied on the solid surfaces for the flow variables $(T, \mathbf{u})$. The boundary condition for the pressure $p$ at the surfaces is zero normal gradient. At the inflow boundary, freestream conditions are maintained throughout the computational process. At the top domain boundary, and the outflow boundary, fluid is allowed to leave the computing region; this condition specifies that the normal gradients of the flow variables $(p, T, \mathbf{u})$ vanish at these boundaries. At the lower boundary in front of and behind the test surfaces below, symmetry condition is applied to all flow variables.

\subsection{Flat plate case setup}

A schematic of the boundary conditions applied in the flat plate cases is shown in Fig. 2. The flow conditions in flat plate experiments [30,31], such as the Mach number, $M a$, freestream

Table 2

Coefficients for gas transport properties.

\begin{tabular}{llllll}
\hline Gas & $A_{S}\left(\mathrm{~Pa} \cdot \mathrm{s} \mathrm{K}^{-1 / 2}\right)$ & $T_{S}(\mathrm{~K})$ & $R\left(\mathrm{~m}^{2} \mathrm{~s}^{-2} \mathrm{~K}^{-1}\right)$ & $\gamma$ & $P r$ \\
\hline Argon & $1.93 \times 10^{-6}$ & 142 & 208.1 & 1.67 & 0.67 \\
Nitrogen & $1.41 \times 10^{-6}$ & 111 & 296.8 & 1.40 & 0.71 \\
\hline
\end{tabular}

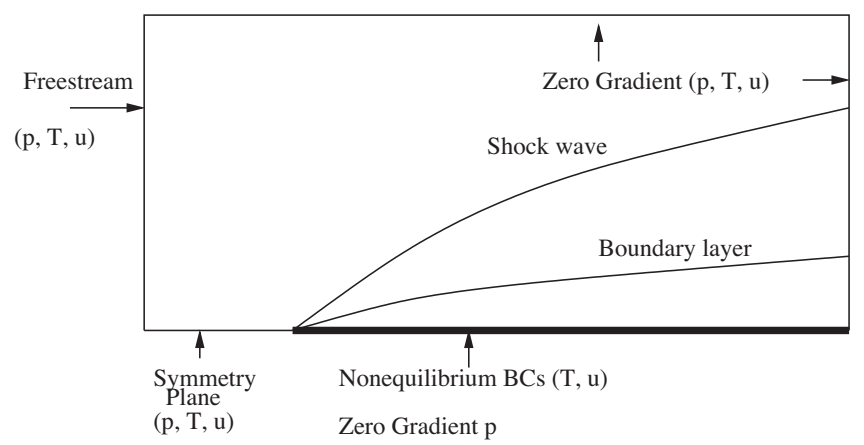

Fig. 2. Numerical setup for the flat plate case. 
temperature, $T_{\infty}$, freestream pressure, $p_{\infty}$ and freestream mean free path, $\lambda_{\infty}$, are shown in Table 3 . A mesh independence analysis was completed to find the final mesh for the convergence solution for all the simulation cases in the present work. Here we only report the final mesh cell sizes. In the flat plate simulations, the computational results are sensitive to the numerical mesh sizes near

Table 3

Flow conditions in the flat-plate experiments.

\begin{tabular}{lrlllll}
\hline Case & \multicolumn{1}{c}{$M a$} & $T_{\infty}(\mathrm{K})$ & $p_{\infty}(\mathrm{Pa})$ & $\lambda_{\infty}(\mathrm{mm})$ & $T_{\mathrm{w}}(\mathrm{K})$ & Gas \\
\hline Becker [30] & 12.7 & 64.5 & 3.73 & 0.23 & 292 & Argon \\
Metcalf et al. [31] & 6.1 & 83.4 & 2.97 & 0.35 & 77 & Nitrogen \\
\hline
\end{tabular}

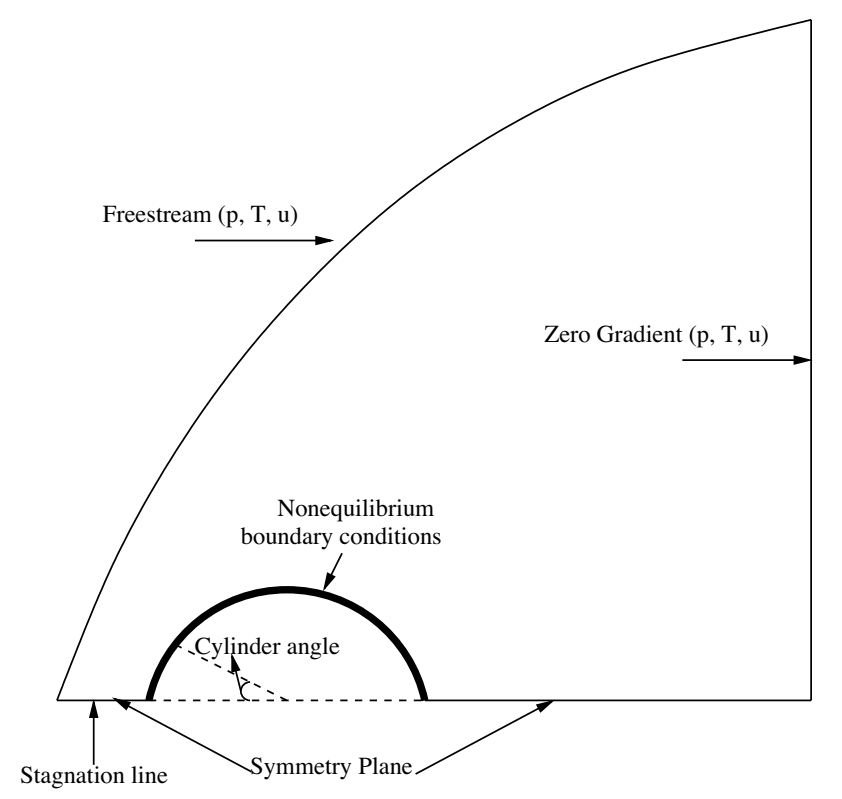

(a) Numerical setup for circular cylinder in cross-flow

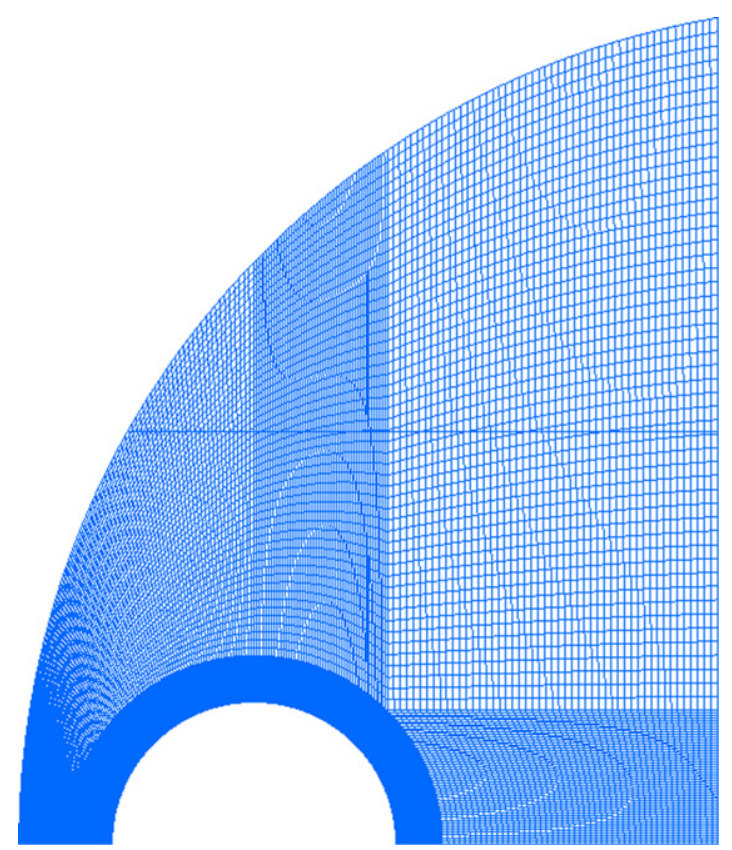

(b) Computational mesh for the cylinder case, with 40,000 cells. the leading edge [29]. A typical mesh for a flat plate simulation is regular rectangular. The final mesh sizes are $\Delta x=\Delta y=0.0767 \mathrm{~mm}$ for Becker's case [30], and $\Delta x=\Delta y=0.117 \mathrm{~mm}$ for Metcalf et al.'s case [31].

\subsection{Circular cylinder in cross-flow case setup}

A schematic of the boundary conditions applied in the cylinder case is shown in Fig. 3(a). The flow conditions for a reported circular cylinder case using the Direct Simulation Monte Carlo (DSMC) technique are as follows $[15,16]$ : freestream conditions $M a=10$, $T_{\infty}=200 \mathrm{~K}, p_{\infty}=1.17 \mathrm{~Pa}, \lambda_{\infty}=3.04 \mathrm{~mm}$, the surface temperature $T_{w}=500 \mathrm{~K}$, diameter of cylinder $D=304.8 \mathrm{~mm}, \mathrm{Kn}=\lambda_{\infty} / D=0.01$, and argon is the working gas. Our computational mesh is constructed to wrap around the leading bow shock; the final mesh encompassing the boundary layer has a linear grading in the surface-normal spacing over the first 25 cells near the surface corresponding to a length of $100 \mathrm{~mm}$, so that the final mesh size varies from 0.05 to $0.25 \mathrm{~mm}$. The mesh has 40,000 cells, and the smallest cell size near the surface is $\Delta x=2.40 \mathrm{~mm}, \Delta y=0.05 \mathrm{~mm}$,

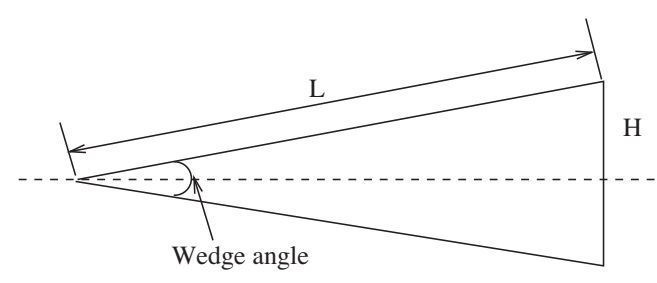

Fig. 4. Geometry of the sharp wedge.

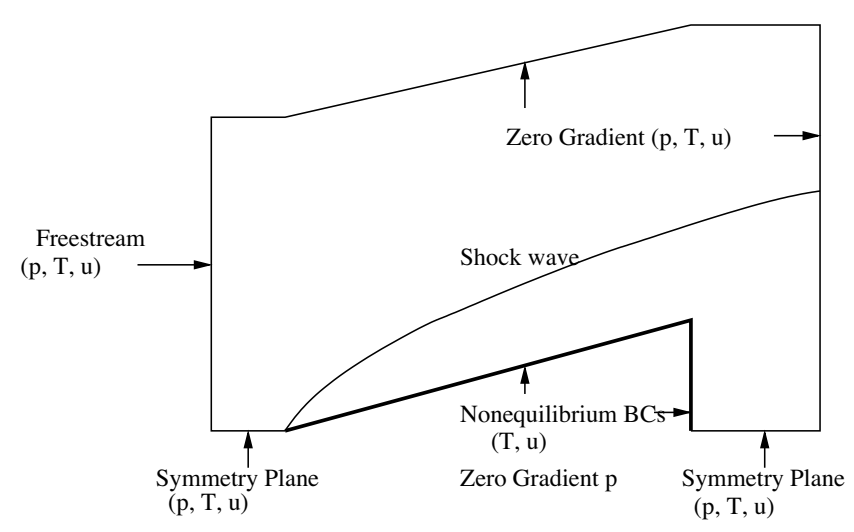

(a) Numerical setup for the sharp wedge

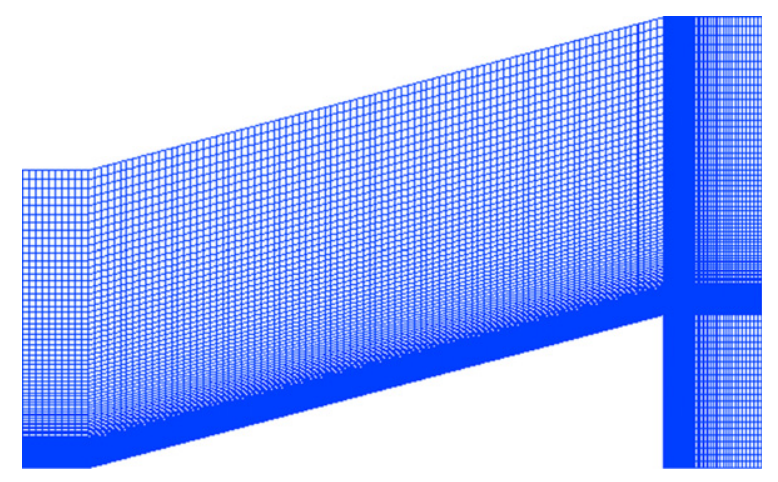

(b) Computational mesh for the sharp case, with 20,000 cells.

Fig. 3. Setup for the circular cylinder in cross-flow case.

Fig. 5. Setup for the sharp wedge case. 
Table 4

Freestream flow conditions of the sharp wedge cases.

\begin{tabular}{llclll}
\hline Case & $p_{\infty}(\mathrm{Pa})$ & $T_{\infty}(\mathrm{K})$ & $M a$ & $T_{\mathrm{w}}(\mathrm{K})$ & Gas \\
\hline McCroskey et al. [32] & 0.76 & 17 & 24.2 & 294 & Nitrogen \\
Lofthouse et al. [16] & 1.17 & 200 & 10 & 500 & Argon \\
\hline
\end{tabular}

Table 5

Geometry and $K n$ of the sharp wedge cases.

\begin{tabular}{llll}
\hline Case & Angle $\left(^{\circ}\right)$ & Height of the base, $\mathrm{H}(\mathrm{mm})$ & $\mathrm{Kn}=\lambda_{\propto} / H$ \\
\hline McCroskey et al. [32] & 30 & 46.4 & 0.002 \\
Lofthouse et al. [16] & 20 & 304.8 & 0.01 \\
\hline
\end{tabular}

which is approximately the same as the smallest cell size in $[15,16]$. A typical mesh of cells for our cylinder simulations is shown in Fig. 3(b).

\subsection{Sharp wedge case setup}

A schematic of the boundary conditions applied in the sharp wedge case is shown in Fig. 5a. The flow conditions and the geometry for representative wedge cases [16,32] are presented in Tables 4 and 5, and in Fig. 4.

Our computational mesh is constructed to encompass the shocks; the final mesh within the boundary layer has a linear grading in the surface-normal spacing. For the McCroskey et al. case [32] this grading occurs over the first 75 cells near the surface, so that our final mesh size varies from $0.05 \mathrm{~mm}$ to $0.5 \mathrm{~mm}$. The smallest cell size near the surface is $\Delta x=1.0 \mathrm{~mm}, \Delta y=0.05 \mathrm{~mm}$, and the mesh has 20100 cells. For the Lofthouse et al. case [15,16] our mesh varies over the first 120 cells near the surface, so that the final mesh size varies from 0.1 to $1.0 \mathrm{~mm}$. The smallest cell size near the surface is $\Delta x=2.2 \mathrm{~mm}, \Delta y=0.1 \mathrm{~mm}$, and the mesh has 88,000 cells. A typical mesh of cells for the sharp wedge simulations is shown in Fig. 5(b).

\section{Results}

Our previous results in [9] showed that the no-slip/jump boundary conditions were unacceptable for simulating thermal gas flows in hypersonics, and so are not investigated in the present work. Our new simulation results for the surface pressure and slip velocity are normalized with the freestream pressure and velocity, respectively. DSMC data for the flat plate cases were generated by using the solver dsmcFoam in OpenFOAM, and DSMC simulations were run with the same freestream conditions and final meshes as the CFD flat plate simulations. As noted in section 2.4, all DSMC data for the circular cylinder case in $[15,16]$, the sharp wedge case in [16] and the flat plate case are obtained with the values $\sigma_{u}=\sigma_{T}=1.0$. All our CFD simulations with the Maxwell/Smoluchowski boundary conditions also used the values $\sigma_{u}=\sigma_{T}=1.0$. The angle of attack is zero for all cases.

\subsection{Metcalf et al. [31] flat plate case}

In the flat plate case, we might choose the characteristic system dimension required for calculating the global $K n$ to be the length of the flat plate; but the plate length has no effect on the slip near its tip. The flat plate case is, in fact, a test case for boundary conditions with a local $K n$ calculated through $x / \lambda=K n^{-1}$ where $x$ is the running distance from the tip of the flat plate. In the figures below, the CFD, DSMC, and experimental results [31] are therefore all plotted against $x / \lambda=K n^{-1}$.

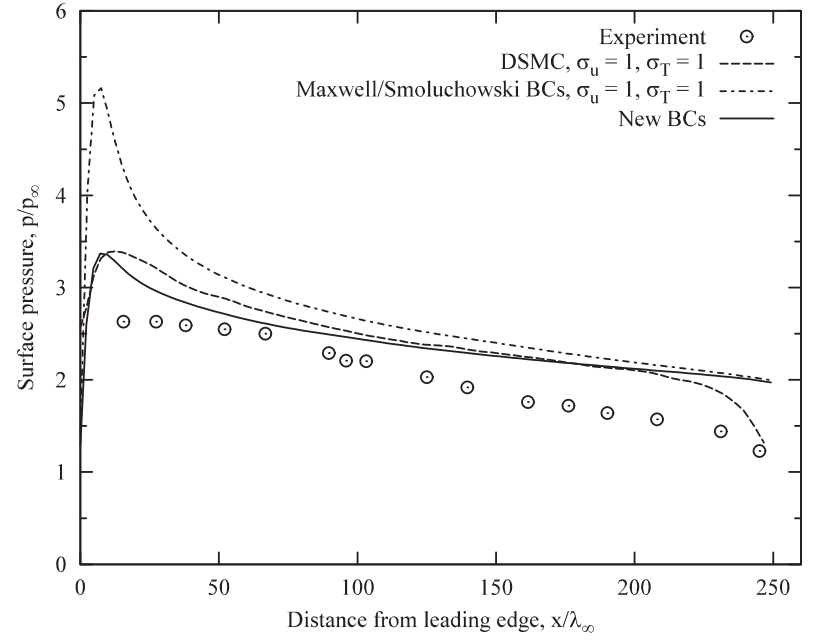

Fig. 6. Metcalf et al. case [31], the surface pressure distribution along the flat plate surface.

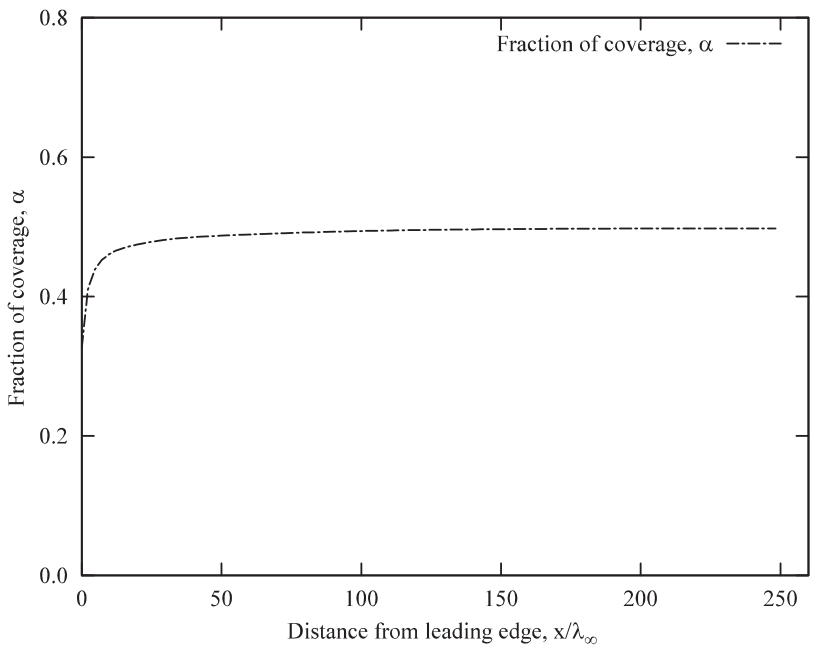

Fig. 7. Metcalf et al. case [31], the distribution of the fraction of gas molecular coverage, $\alpha$, along the flat plate surface.

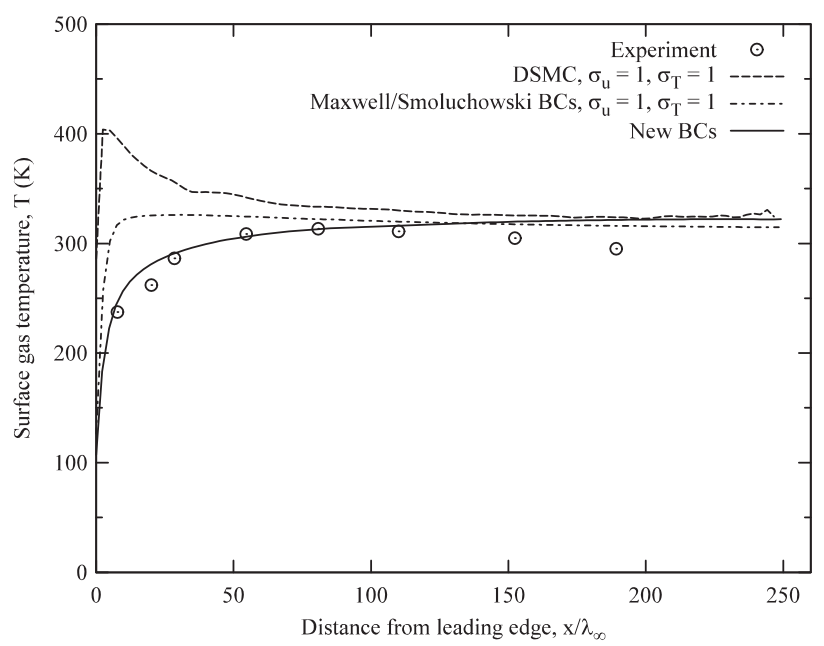

Fig. 8. Metcalf et al. case [31], the surface gas temperature along the flat plate surface. 


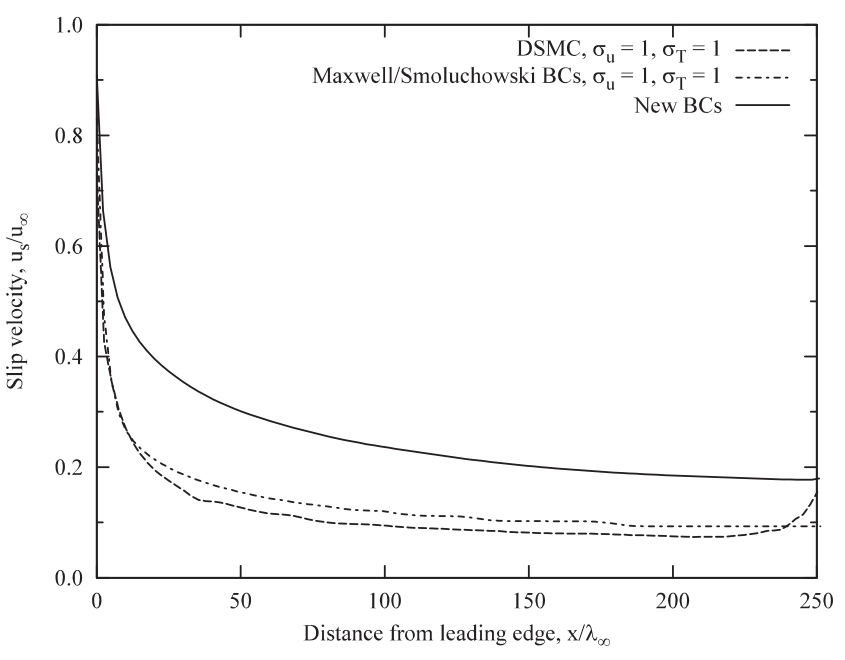

Fig. 9. Metcalf et al. case [31], the slip velocity along the flat plate surface.

For the surface pressure, the conventional Maxwell/Smoluchowski conditions generally do not give good agreement with experimental data, as seen in Fig. 6. The results of the DSMC data, and our CFD using the new boundary conditions, are close together and give relatively good agreement with the experimental data [31] for $x / \lambda_{\infty} \geqslant 25$ (i.e. $K n \leqslant 0.04$ ). From the plate tip, the fraction $\alpha$ increases from 0.35 to 0.47 at the location $x / \lambda_{\infty}=30$, and then stays nearly constant along the flat plate, as seen in Fig. 7.

Considering the surface gas temperature in Fig. 8, the DSMC data and the results with the Maxwell/Smoluchowski conditions do not give good agreement with experimental data towards the tip of the flat plate. By comparison, the results using the new boundary conditions give the best agreement with the experimental data.

For slip velocity, the DSMC data are close to the results of the Maxwell/Smoluchowski conditions. The results from these two cases are lower than the results using our new boundary conditions, as seen in Fig. 9.

\subsection{Becker's flat plate case [30]}

In Becker's case, surface pressure data (Fig. 10) shows that the results using the new boundary conditions are in good agreement

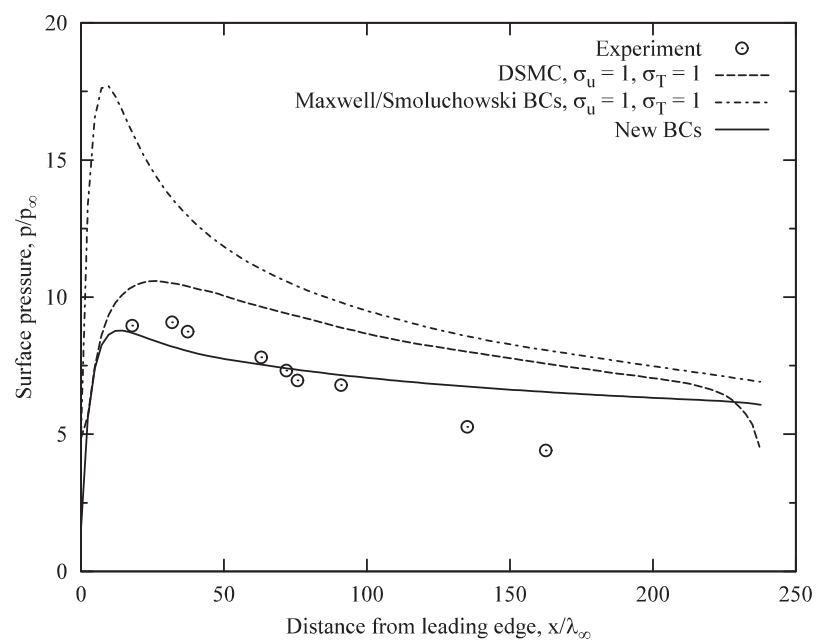

Fig. 10. Becker's case [30], the surface pressure distribution along the flat plate surface.

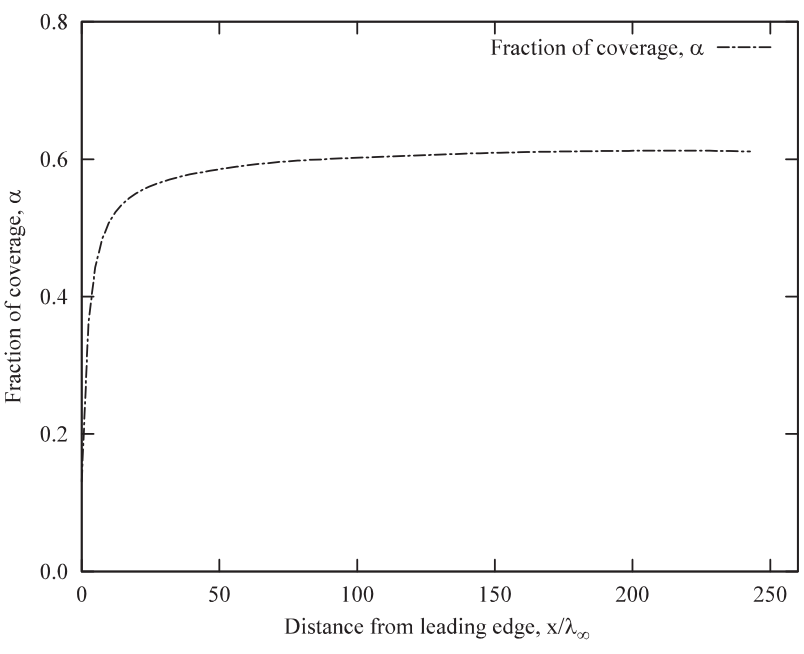

Fig. 11. Becker's case [30], the distribution of the fraction of gas molecular coverage, $\alpha$, along the flat plate surface.

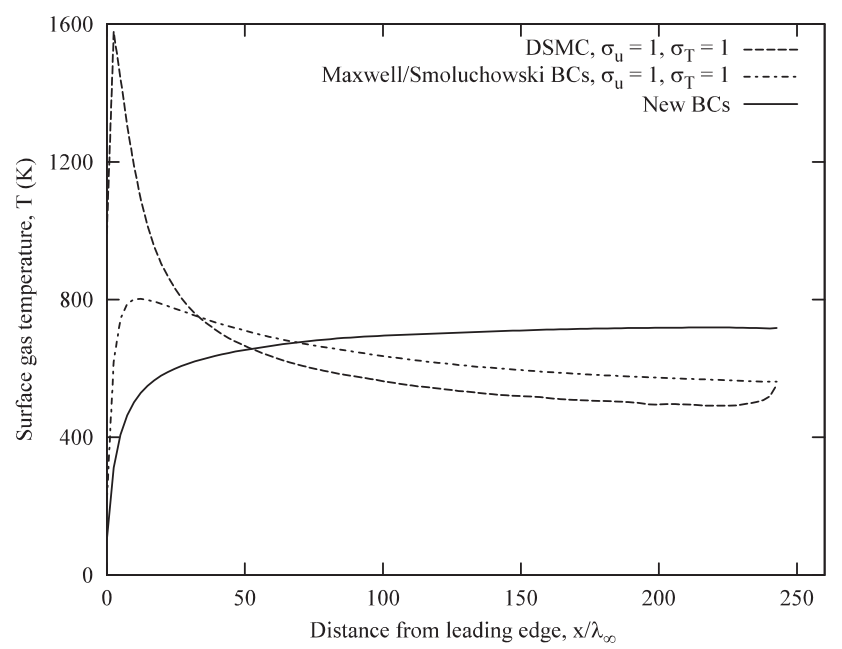

Fig. 12. Becker's case [30], the surface gas temperature along the flat plate surface.

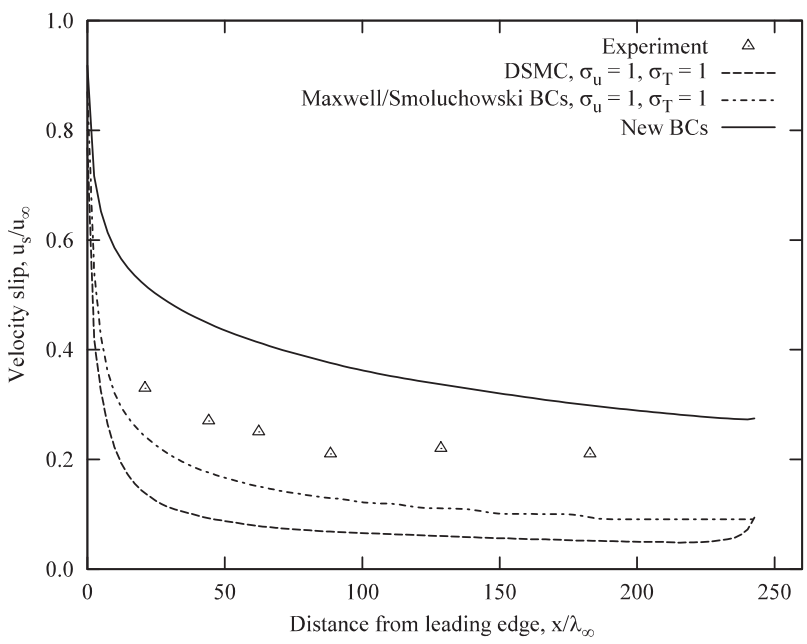

Fig. 13. Becker's case [30], the slip velocity along the flat plate surface. 
with experimental data, and provide much better results than the Maxwell/Smoluchowski conditions. On the other hand, the DSMC data are not in particularly good agreement with experiment. From the plate tip, the fraction $\alpha$ increases from 0.17 to 0.57 by the location $x / \lambda_{\infty}=50$, and then stays nearly constant along the flat plate, as seen in Fig. 11.

In Fig. 12 for the surface gas temperature, at the tip of the flat plate there is a substantial difference between the results of the Maxwell/Smoluchowski conditions and the CFD results using the new boundary conditions, as well as the DSMC data.

Fig. 13 for slip velocity shows that the DSMC data do not agree well with the experimental data, giving the lowest slip value of any of the simulations. The Maxwell/Smoluchowski conditions underpredict the slip velocity, while the new boundary conditions predict a higher slip velocity than the experimental data.

\subsection{Circular cylinder in cross-flow $[15,16]$}

In this case, our new slip condition is tested both with and without the curvature effect (i.e. the terms in $\Pi_{\mathrm{mc}}$ in Eq. (25)). Considering the surface pressure, all the CFD simulations give good agreement with the DSMC data reported in $[15,16]$, as seen in Fig. 14. The fraction $\alpha$ is nearly constant in the region $0^{\circ} \leqslant \Phi \leqslant 90^{\circ}$ and then decreases towards the location $\Phi=135^{\circ}$, where $\Phi$ is the cylinder angle. It then starts to rise towards $\Phi=165^{\circ}$, and then stays nearly constant over $165^{\circ} \leqslant \Phi \leqslant 180^{\circ}$ (Fig. 15). There is no dependence of the values of $\alpha$ on whether the curvature effect is included or not.

Considering the temperature jump in Fig. 16, all the CFD results predict a higher temperature jump than the DSMC data in $[15,16]$ for cylinder angles $0^{\circ} \leqslant \Phi \leqslant 100^{\circ}$, and the new conditions give the highest predictions. The gas flow begins to expand between $110^{\circ} \leqslant \Phi \leqslant 130^{\circ}$, and all the CFD temperature jumps decrease while the DSMC predicted temperature jump increases. At the location of the separation point (around $\Phi=130^{\circ}$ ), the gas flow begins to enter the wake region. Here all the CFD temperature jumps rise towards $\Phi=170^{\circ}$ and are nearly constant between $170^{\circ} \leqslant \Phi \leqslant 180^{\circ}$. However, the DSMC predicted temperature jump decreases to the location $\Phi=170^{\circ}$ and is nearly constant between $170^{\circ} \leqslant \Phi \leqslant 180^{\circ}$. There are large differences between the CFD temperature jumps and the DSMC temperature jump between $150^{\circ} \leqslant \Phi \leqslant 180^{\circ}$. These differences may be understood by the fact that the translational temperature jump in the DSMC method is calculated by the components of gas velocity and the slip velocity only in [15,16], while the translational temperature jump in the CFD method is calculated by

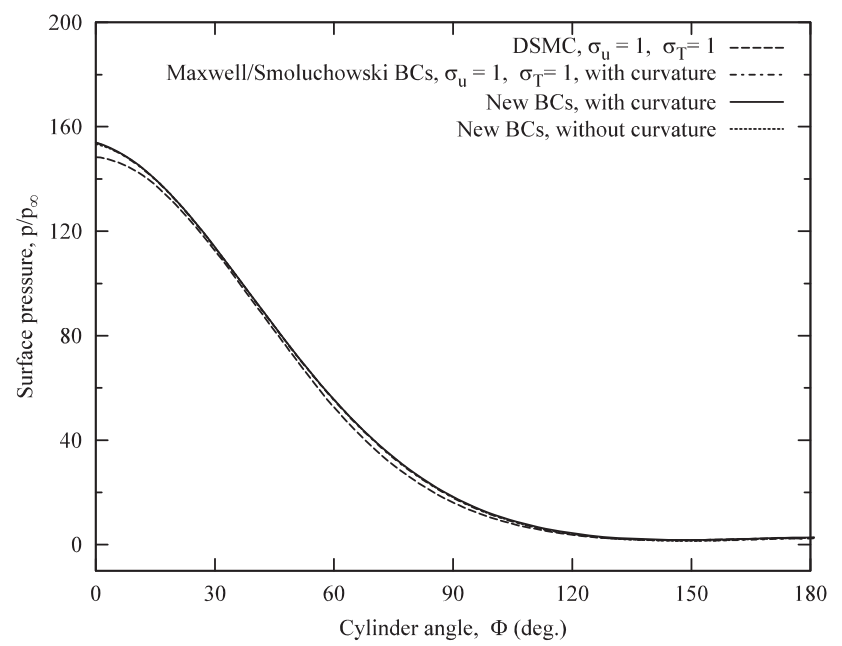

Fig. 14. Cylinder case $[15,16]$, surface pressure distribution around the cylinder surface, $K n=0.01$.

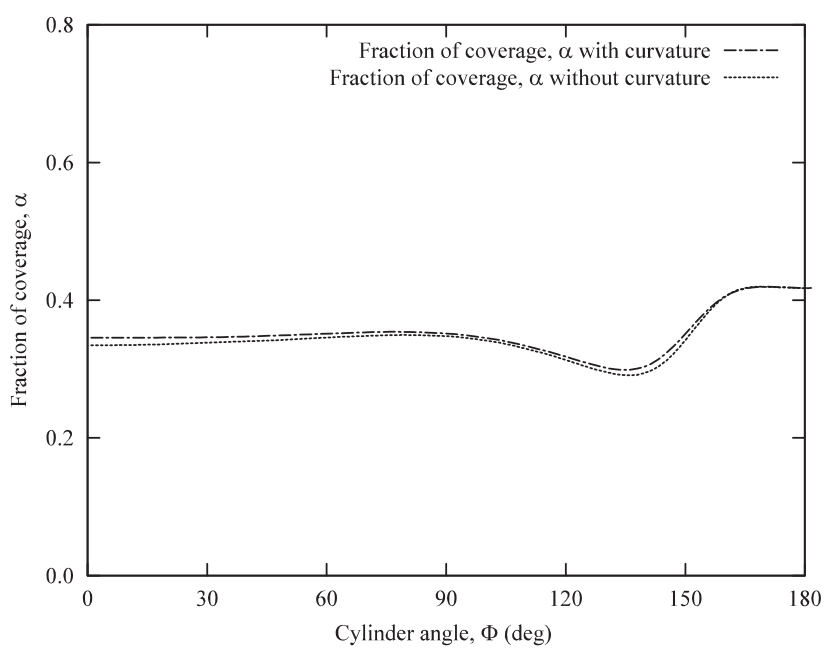

Fig. 15. Cylinder case $[15,16]$, the distribution of the fraction of gas molecular coverage, $\alpha$, around the cylinder surface, $\mathrm{Kn}=0.01$.

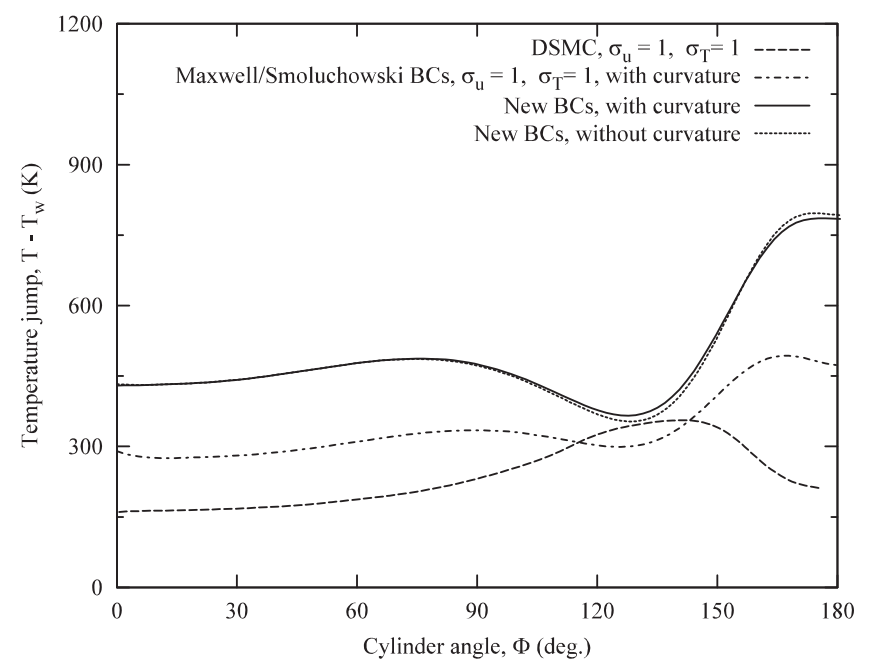

Fig. 16. Cylinder case $[15,16]$, temperature jump distribution around the cylinder surface, $K n=0.01$.

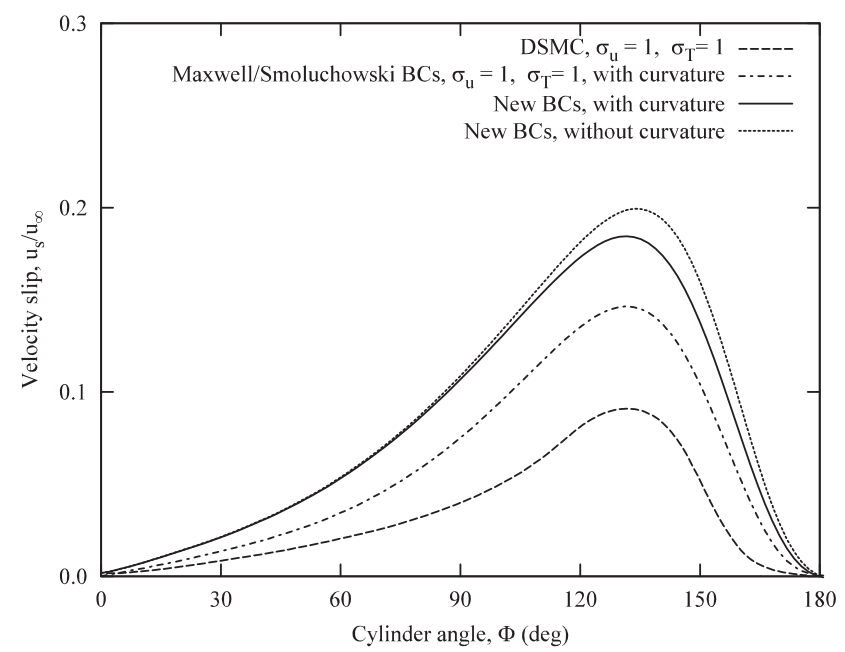

Fig. 17. Cylinder case $[15,16]$, slip velocity distribution around the cylinder surface, $K n=0.01$. 
the normal gradient of gas temperature, and is independent of the gas velocity. This makes the profile of the DSMC temperature jump similar to that for the slip velocity, as seen by comparison between Figs. 16 and 17.

All our CFD tests predict a higher slip velocity than the DSMC data given in $[15,16]$, as seen in Fig. 17. The DSMC and CFD slip velocities increase gradually between $0^{\circ} \leqslant \Phi \leqslant 135^{\circ}$, reaching peak normalized values around the location $\Phi=135^{\circ}$ as follows: (a) 0.145 using the Maxwell/Smoluchowski conditions with the curvature effect and $\sigma_{u}=\sigma_{T}=1.0$, (b) 0.18 and 0.20 for the new boundary conditions with and without the curvature effect, respectively, and (c) 0.09 for the DSMC data. The curvature effect reduces the peak value of the slip velocity. However, the curvature effect in the new slip condition does not affect the temperature jump or the surface pressure, as seen in Figs. 14 and 16.

\subsection{Flow over a sharp wedge [16]}

The simulation results for the wedge cases are plotted as a function of the distance $S$ along the wedge surface, normalized by the length $L$ of the top surface. Therefore $S / L=1$ is the location of the wedge shoulder and the beginning of the wake.

Considering the surface pressures in Fig. 18, the results with our new conditions are in good agreement with the DSMC data in [16] before the wedge shoulder. The Maxwell/Smoluchowski conditions, however, overpredict the surface pressures at the leading edge. In the region $1.0 \leqslant S / L \leqslant 1.17$ all the CFD results are close together and there is a slight difference with the DSMC data.

The fractions, $\alpha$, with and without the curvature effect included in the new slip condition, are the same (Fig. 19). They reach a peak value of 0.48 at the tip, and then decrease until the wedge shoulder. Then $\alpha$ gradually increases in the region $1.05 \leqslant S / L \leqslant 1.15$.

The temperature jumps in the CFD results and the DSMC data in [16] are highest at the leading edge, as seen in Fig. 20, with a peak temperature jump of (a) about $1150 \mathrm{~K}$ with the new conditions, (b) about $1250 \mathrm{~K}$ when using the Maxwell/Smoluchowski conditions, and (c) about $2129 \mathrm{~K}$ in the DSMC simulation. Past the leading edge, the temperature jumps gradually decrease along the surface and, in addition, show a slight bump around the wedge shoulder. In the region $0.1 \leqslant S / L \leqslant 0.95$ the new conditions predict a higher temperature jump than that given by the Maxwell/Smoluchowski conditions and the DSMC data. In the region $1.0 \leqslant S / L \leqslant 1.17$, all CFD results are quite close to the DSMC data.

Fig. 21 shows that, at the leading edge, both the CFD results and the DSMC data in [16] show a peak normalized slip velocity of (a) about 0.79 for the new conditions with and without the curvature

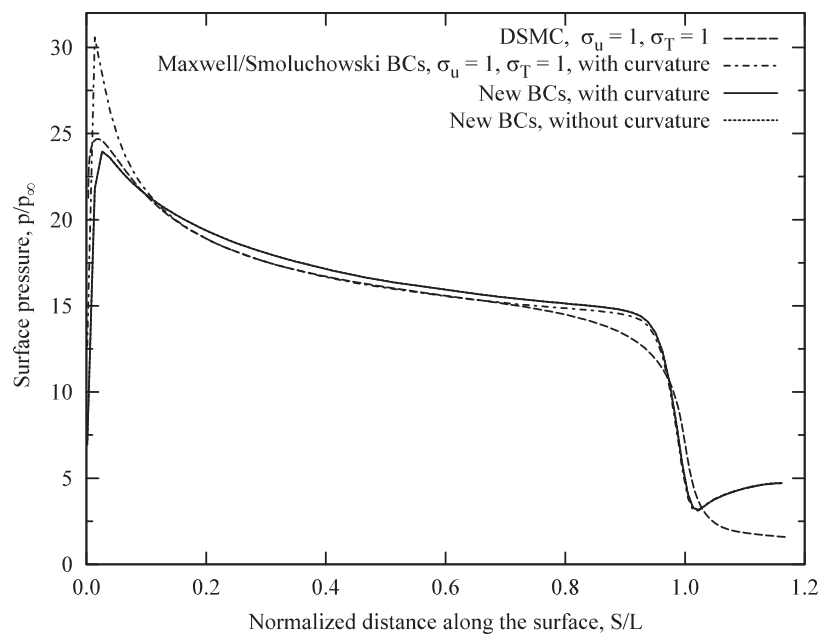

Fig. 18. Wedge case [16], pressure distribution along the wedge surface, $K n=0.01$.

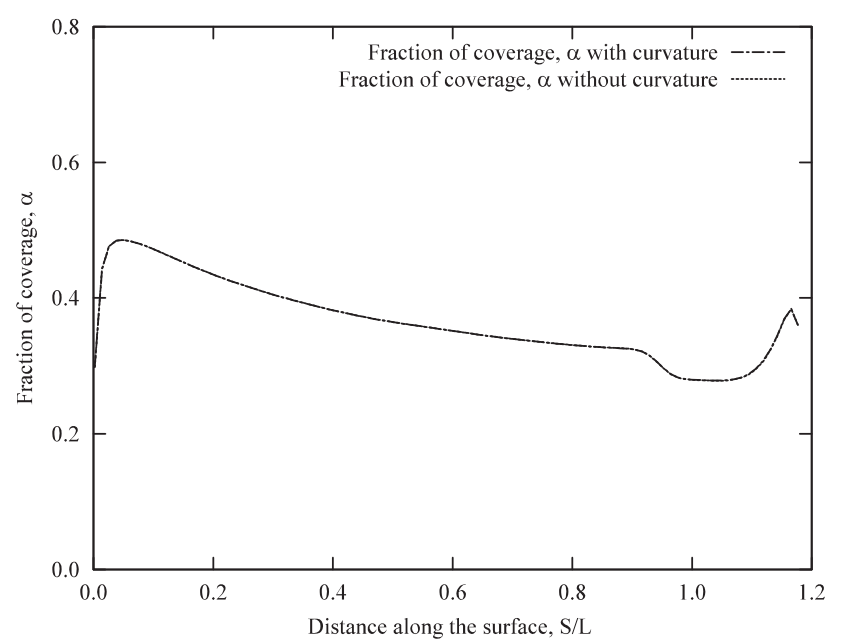

Fig. 19. Wedge case [16], the distribution of the fraction of gas molecular coverage, $\alpha$, along the wedge surface, $K n=0.01$.

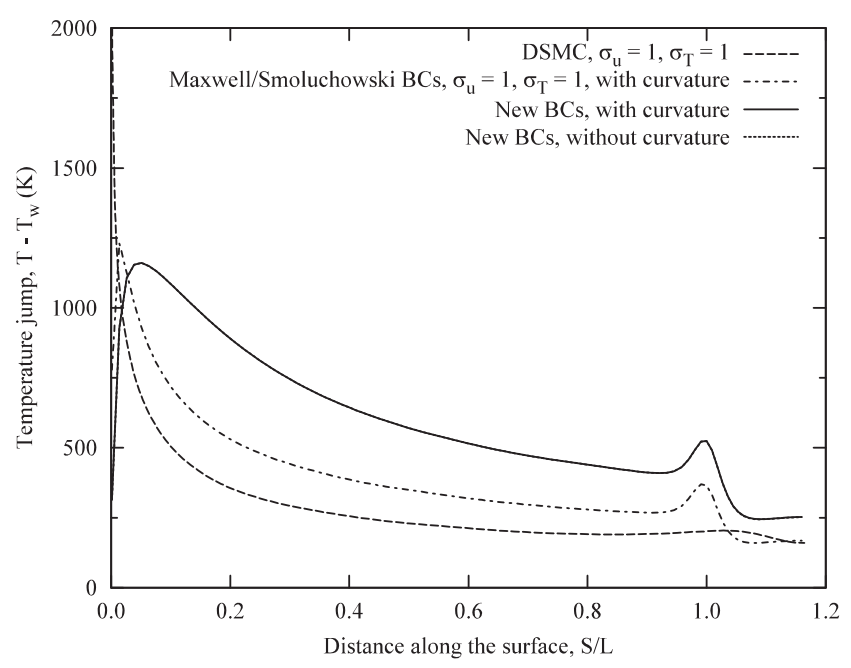

Fig. 20. Wedge case [16], temperature jump distribution along the wedge surface, $K n=0.01$.

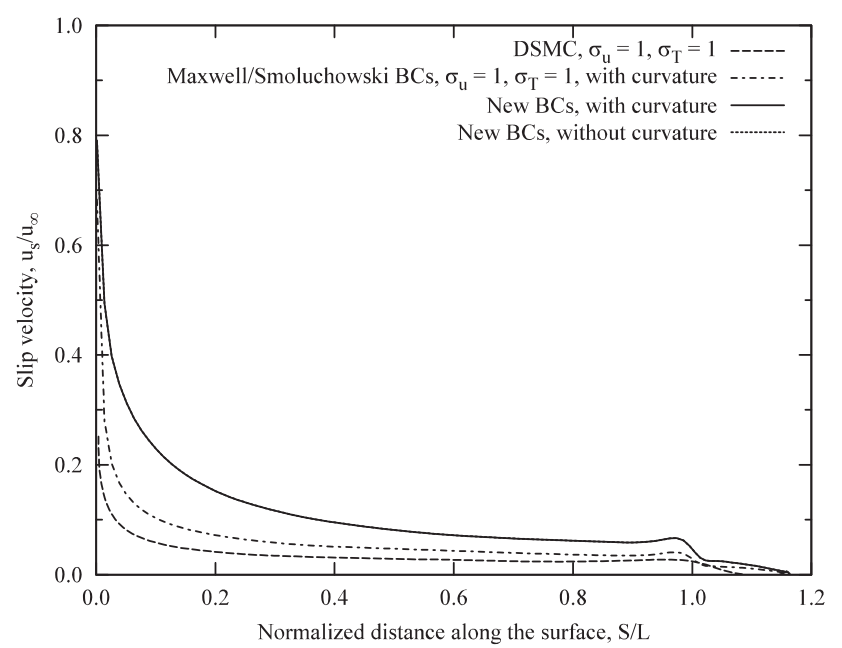

Fig. 21. Wedge case [16], slip velocity distribution along the wedge surface, $K n=0.01$. 
effect, (b) about 0.68 when using the Maxwell/Smoluchowski conditions with the curvature effect, and (c) about 0.25 for the DSMC simulation. Past the leading edge, the slip velocities very quickly reduce to a nearly constant value until the wedge shoulder; after the shoulder the DSMC data are lower than all the CFD results. The slip velocities when using the new conditions are higher than both the slip velocities of the simulation with the Maxwell/Smoluchowski conditions and the DSMC data until the wedge shoulder is reached. On the surface of the base of the wedge $(1.0 \leqslant S / L \leqslant 1.17)$, all the CFD results are close to the DSMC data.

The curvature effect in the new slip condition does not affect the surface pressure or surface as temperature in this case. It is seen that the sharp wedge case is equivalent to the flat plate case with an angle of attack as half the wedge angle.

\subsection{McCroskey et al. [32] wedge case}

In this case, only the results of the surface pressure are presented because there are no experimental or DSMC data for the slip velocity or the temperature jump reported in the literature.

Considering the surface pressures, Fig. 22 shows the new boundary conditions give results in best agreement with the experimental data. At the tip of the wedge, and similar to the flat plate case, the

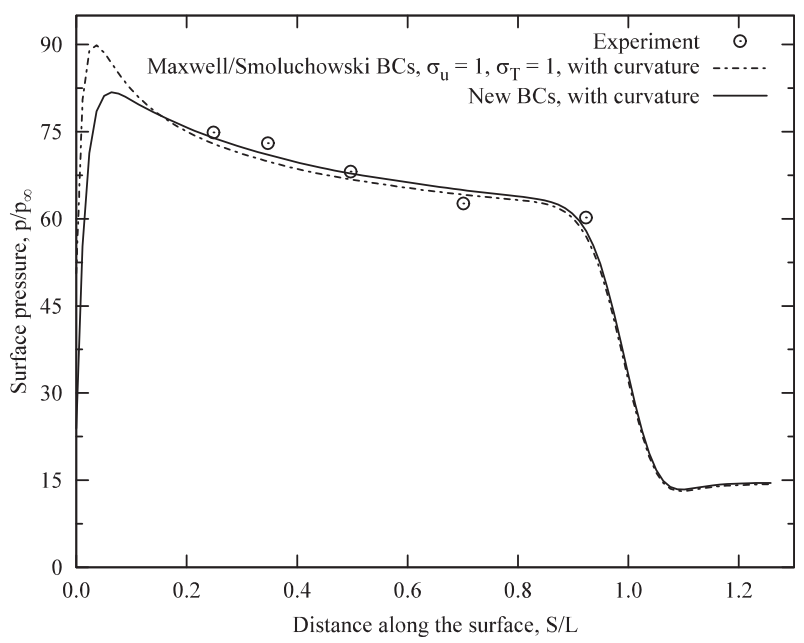

Fig. 22. McCroskey et al. wedge case [32], surface pressure distribution along the wedge surface, $K n=0.002$.

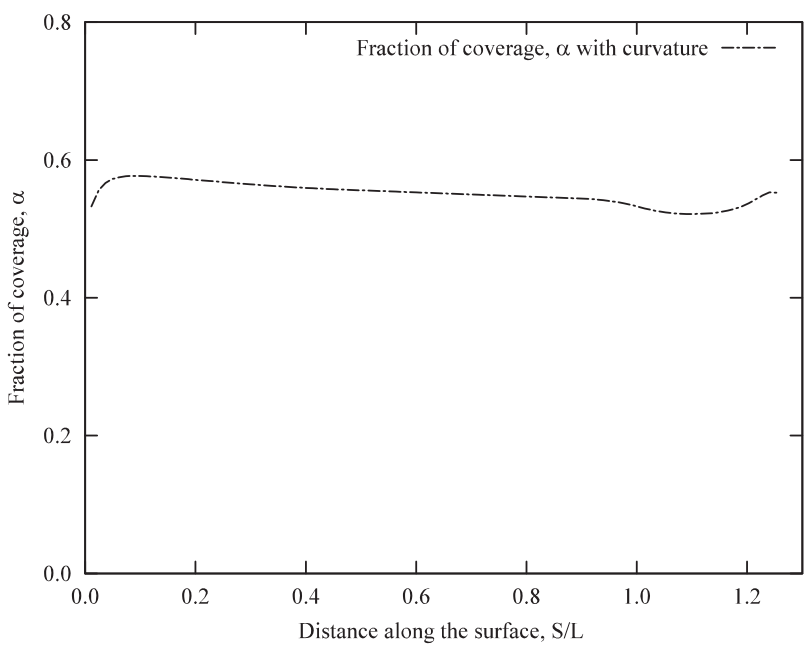

Fig. 23. McCroskey et al. wedge case, the distribution of the fraction of gas molecular coverage, $\alpha$, along the wedge surface, $K n=0.002$. new conditions predict a lower peak normalized surface pressure than given by the Maxwell/Smoluchowski conditions. All simulation results are close together along the base $(1 \leqslant S / L \leqslant 1.2)$. In Fig. 23 the fraction $\alpha$ reaches a peak value of 0.58 at $S / L=0.05$, and then slightly decreases until location $S / L=1.05$. It slightly rises in the region $1.15 \leqslant S / L \leqslant 1.25$.

\section{Conclusions}

For sharp geometries, such as the sharp-leading-edge flat plate and the sharp wedge, the new boundary conditions derived in the present work give better predictions, compared to both experimental and DSMC data, for the surface pressure [16,30-32], and the surface gas temperature [31]. Overall, our new boundary conditions predict a higher slip velocity than do the Maxwell/Smoluchowski conditions (with $\sigma_{u}=\sigma_{T}=1.0$ ) and DSMC.

Considering blunt geometries, such as the circular cylinder, the new boundary conditions give good agreement with DSMC data $[15,16]$ for the surface pressure. They predict higher temperature jumps and slip velocities than the Maxwell/Smoluchowski conditions and DSMC do. In the region $150^{\circ} \leqslant \Phi \leqslant 180^{\circ}$, there is a large difference in predictions of the temperature jump between the CFD and the DSMC. The curvature effect does have an effect on the predicted slip velocity in this case.

In the design of aerospace vehicles, a precise prediction of gas surface temperature, though desirable, may not be essential, in that the thermal protection will be designed to withstand much higher temperatures than are predicted. However, an accurate prediction of drag, as obtained through the surface pressure distribution, is important for a complete understanding of the flight vehicle dynamics and an accurate prediction of the vehicle's trajectory [16]. The new boundary conditions proposed here are based on a combination of the Langmuir adsorption isotherm and the Maxwell/Smoluchowski conditions. They give somewhat better predictions for surface pressures, which would then lead to better predictions for aerodynamic drag. These new conditions go some way towards addressing the problem of the values of free parameters, such as the tangential momentum $\sigma_{u}$, and the thermal $\sigma_{T}$, accommodation coefficients in the first order slip/jump conditions. The fraction $\alpha$ is not constant along the solid surface for all the CFD simulation cases. This is more realistic than applying constant values of $\sigma_{u}$ and $\sigma_{T}$ along the solid surface, which is usually done in CFD simulations. Our new conditions do, however, predict higher surface gas temperatures than both the Maxwell/Smoluchoski conditions and DSMC in the circular cylinder case.

\section{Acknowledgements}

N.T.P.L. would like to thank the James Weir Foundation and the University of Strathclyde for research scholarships that supported this work. He also wishes to thank the National Research Foundation of Korea for partial support provided under Grant No. 20110031383. R.S.M. wishes to acknowledge partial support from the Degree and Research Center for Aerospace Green Technology (DRC) of the Korea Aerospace Research Institute (KARI), funded by the Korea Research Council of Fundamental Science \& Technology (KRCF).

\section{References}

[1] J.C. Maxwell, On stresses in rarefied gases arising from inequalities of temperature, Phil. Trans. Roy. Soc. 11 (170) (1879) 231-256.

[2] S. Shen, G. Chen, R.M. Crone, M. Anaya-Dufresne, A kinetic theory based first order slip boundary condition for gas flow, Phys. Fluids 19 (2007) 086101.

[3] A.Q. Zade, M. Renksizbulut, J. Friedman, Boundary conditions for multicomponent slip-flows based on the kinetic theory of gases, Proc. of ASME ICNMM2008, vol. 80365, Darmstadt, Germany, 2008. 
[4] T. Gökçen, R.W. MacCormack, Nonequilibrium effects for hypersonic transitional flows using continuum approach, AIAA Paper (1989) 0461-1989.

[5] D.A. Lockerby, J.M. Reese, D.R. Emerson, R.W. Barber, Velocity boundary condition at solid walls in rarefied gas calculations, Phys. Rev. E 70 (2004) 017303.

[6] R.G. Deissler, An analysis of second order slip flow and temperature jump boundary conditions for rarefied gases, Int. J. Heat Mass Transfer 7 (1964) 681694.

[7] N. G Hadjiconstantinou, Comment on Cercignani's second order slip coefficient, Phys. Fluids 15 (2003) 2352-2354.

[8] C. Cercignani, Mathematical Methods in Kinetic Theory, Plenum, New York 1969.

[9] N. T. P. Le, C. J. Greenshields, J. M. Reese, Evaluation of nonequilibrium boundary conditions for hypersonic rarefied gas flows, in: Proceedings of the third European Conference for AeroSpace Sciences, EUCASS2009 86, 2009.

[10] M. von Smoluchowski, Über wärmeleitung in verdünnten gasen, Annalen der Physik und Chemie 64 (1898) 101-130.

[11] R.S. Myong, Gaseous slip models based on the Langmuir adsorption isotherm, Phys. Fluids 16 (2004) 104-117.

[12] R.S. Myong, J.M. Reese, R.W. Barber, D.R. Emerson, Velocity slip in microscale cylindrical Couette flow: the Langmuir model, Phys. Fluids 17 (2005) 087105.

[13] G.N. Patterson, Molecular Flow of Gases, Wiley, New York, 1956.

[14] E.H. Kennard, Kinetic Theory of Gases, McGraw-Hill Book Company Inc., 1938.

[15] A. Lofthouse, L.C. Scalabrin, I.D. Boyd, Velocity slip and temperature jump in hypersonic aerothermodynamics, J. Thermophys. Heat Transfer 22 (1) (2008) 38-49.

[16] A. J. Lofthouse, Nonequilibrium hypersonic aerothermodynamics using the direct simulation Monte Carlo and Navier-Stokes models, PhD Thesis, University of Michigan, 2008.

[17] D.K. Bhattacharya, B.C. Eu, Nonlinear transport processes and fluid dynamics: effects of thermoviscous coupling and nonlinear transport coefficients on plane Couette flow of Lennard-Jones fluids, Phys. Rev. A 35 (2) (1987) 821-836.

[18] H. Choi, D.H. Lee, D. Lee, Complex microscale flow simulations using langmuir slip condition, Numer. Heat Transfer A 48 (2005) 407-425.
[19] R.S. Myong, D. A Lockerby, J. M Reese, The effect slip on microscale heat transfer: an extended Graetz problem, J. Heat Mass Transfer Int. 49 (2006) 2502-2513.

[20] S. Dushman, Scientific Foundations of Vacuum Techniques, Wiley, New York, 1962.

[21] B. Cordero, V. Gómez, A.E. Platero-Prats, M. Revés, J. Echeverría, E. Cremades, F. Barragán, S. Alvarez, Covalent radii revisited, Dalton Trans. (2008) 2832-2838.

[22] R.I. Masel, Principles of Adsorption and Reaction on Solid Surfaces, John Wiley \& Sons, New York, 1996.

[23] J. Tóth, Adsorption: Theory, Modeling and Analysis, Surfactant Science Series 107 (2001).

[24] www.openfoam.com 2010.

[25] C.J. Greenshields, H.G. Weller, L. Gasparini, J.M. Reese, Non-oscillatory central schemes for high speed viscous flows, Int: J. Numer. Methods Fluids 23 (2010) $1-21$.

[26] C.R. Lilley, M.N. Macrossan, DSMC calculations of shock structure with various viscosity laws, in: Proceedings of the twenty third International Symposium on Rarefied Gas Dynamics (2002) pp. 663-675.

[27] R.C. Weast, Chemical Rubber Company (CRC), CRC Handbook of Chemistry and Physics, CRC Press, Inc., Florida, 1984.

[28] Crane Company, Flow of fluid through valves, fittings and pipes, Technical Paper No. 410, 1988.

[29] N.T.P. Le, Nonequilibrium boundary conditions for the Navier-Stokes-Fourier equations in hypersonic gas flow simulations, $\mathrm{PhD}$ Thesis, University of Strathclyde, 2010.

[30] M. Becker, Flat plate flow files and surface measurements from merged layer into transition regime, Proceedings of the seventh International Symposium on Rarefied Gas Dynamics (1969) pp. 515-528.

[31] S.C. Metcalf, D.C. Lillicrap, C. J. Berry, A study of the effect of surface temperature on the shock-layer development over sharp-edge shapes in lowReynolds-number high-speed flow, Proceedings of the seventh International Symposium on Rarefied Gas Dynamics (1969) pp 619-634.

[32] W.J. McCroskey, S.M. Bogdonoff, A.P. Genchi, Leading edge flow studies of sharp bodies in rarefied hypersonic flows, Proceedings of the fifth International Symposium on Dynamics (1967) pp. 1047-1066. 